\title{
Oculogastrointestinal muscular dystrophy
}

INSERM

\section{Source}

INSERM. (1999). Orphanet: an online rare disease and orphan drug data base.

Oculogastrointestinal muscular dystrophy. ORPHA:1876

Oculogastrointestinal muscular dystrophy is an extremely rare autosomal recessively inherited neuromuscular disease characterized by ocular manifestations such as ptosis and diplopia followed by chronic diarrhea, malnutrion and intestinal peudo-obstruction. 